\title{
Early Diagnosis of Helicobacter pylori Infection in Vietnamese Patients with Acute Peptic Ulcer Bleeding: A Prospective Study
}

\author{
Duc Trong Quach, ${ }^{1,2}$ Mai Ngoc Luu, ${ }^{1,2}$ Toru Hiyama, ${ }^{3}$ \\ Thuy-HuongThi To, ${ }^{4}$ Quy Nhuan Bui, ${ }^{4}$ Tuan Anh Tran, ${ }^{4}$ Binh Duy Tran, \\ Minh-Cong Hong Vo, ${ }^{2}$ Shinji Tanaka, ${ }^{5}$ and Naomi Uemura ${ }^{6}$ \\ ${ }^{1}$ Department of Internal Medicine, University of Medicine and Pharmacy, Ho Chi Minh, Vietnam \\ ${ }^{2}$ Department of Gastroenterology, Gia Dinh People's Hospital, Ho Chi Minh City, Vietnam \\ ${ }^{3}$ Health Service Center, Hiroshima University, Higashihiroshima, Japan \\ ${ }^{4}$ Department of Endoscopy, Gia Dinh People's Hospital, Ho Chi Minh City, Vietnam \\ ${ }^{5}$ Department of Endoscopy, Hiroshima University Hospital, Hiroshima, Japan \\ ${ }^{6}$ Department of Gastroenterology and Hepatology, National Center for Global Health and Medicine, Ichikawa, Japan
}

Correspondence should be addressed to Toru Hiyama; tohiyama@hiroshima-u.ac.jp

Received 25 September 2016; Accepted 21 November 2016; Published 4 January 2017

Academic Editor: Ford Bursey

Copyright (c) 2017 Duc Trong Quach et al. This is an open access article distributed under the Creative Commons Attribution License, which permits unrestricted use, distribution, and reproduction in any medium, provided the original work is properly cited.

\begin{abstract}
Aims. To investigate $H$. pylori infection rate and evaluate a combined set of tests for $H$. pylori diagnosis in Vietnamese patients with acute peptic ulcer bleeding (PUD). Methods. Consecutive patients with acute PUB were enrolled prospectively. Rapid urease test (RUT) with 3 biopsies was carried out randomly. Patients without RUT or with negative RUT received urea breath test (UBT) and serological and urinary $H$. pylori antibody tests. $H$. pylori was considered positive if RUT or any noninvasive test was positive. Patients were divided into group A (RUT plus noninvasive tests) and group B (only noninvasive tests). Results. The overall $H$. pylori infection rate was $94.2 \%$ (161/171). Groups A and B had no differences in demographic characteristics, bleeding severity, endoscopic findings, and proton pump inhibitor use. H. pylori-positive rate in group A was significantly higher than that in group B (98.2\% versus $86.7 \%, p=0.004)$. The positive rate of RUT was similar at each biopsy site but significantly increased if RUT results from 2 or 3 sites were combined $(p<0.05)$. Conclusions. H. pylori infection rate in Vietnamese patients with acute PUB is high. RUT is an excellent test if at least 2 biopsies are taken.
\end{abstract}

\section{Introduction}

Helicobacter pylori (H. pylori) is one of the leading causes of peptic ulcer disease, which may lead to severe complications such as peptic ulcer bleeding (PUB) or perforation. Diagnosis and successful $H$. pylori eradication have been shown to prevent recurrent PUB $[1,2]$. Recently, the importance of early diagnosis and eradication of $H$. pylori during admission period of patients with PUB has been further emphasized because the rate of recurrent PUB has been reduced with such strategy [3]. A recent study reported that up to $40 \%$ of PUB patients with $H$. pylori infection did not receive $H$. pylori eradication therapy and were lost to follow-up if diagnostic tests were performed after discharge [4]. Another study showed that less than $50 \%$ of patients with PUB received $H$. pylori testing and less than $10 \%$ had any $H$. pylori testing after discharge [5].

The fact that many diagnostic tests for $H$. pylori are not so highly sensitive and have high false-negative value during acute bleeding period creates a clinical challenge [68]. As a consequence, the diagnosis of $H$. pylori infection during acute bleeding situations has been reported to be lower than the true number [9]. A combination of diagnostic tests may help to document more correctly the prevalence of $H$. pylori infection among patients with PUB. The rates of $H$. pylori infection among Vietnamese patients with upper gastrointestinal symptoms were reported of $55.5 \%-65.5 \%$ in previous studies $[10,11]$. But there have been no studies 
on the prevalence of $H$. pylori in Vietnamese patients with acute PUB. The Maastricht IV consensus recommended that $H$. pylori eradication treatment should be started at reintroduction of oral feeding in cases of bleeding ulcer as delaying treatment after discharge leads to reduced compliance or loss to follow-up without receiving treatment [12]. In addition, validated IgG antibody can be used to diagnose H. pylori infection in patients with no prior history of $H$. pylori eradication. In clinical practice, therefore, only one positive test is enough to initiate $H$. pylori eradication therapy in patients with bleeding peptic ulcers. In this study, we imitate the real clinical scenario of peptic ulcer bleeding, when sometimes invasive diagnostic tests for $H$. pylori (rapid urease test, histology, and culture) could not be done. This study aimed to assess the prevalence of $H$. pylori infection and evaluate the role of a combined set of tests for $H$. pylori early diagnosis in Vietnamese patients with acute PUB. We did not assess the sensitivity and specificity of each diagnostic test, which has been very nicely reported in a previous metaanalysis [7], but try to identify the testing strategy which has the high possibility of early detecting $H$. pylori infection in this setting.

\section{Methods}

2.1. Patients. From August 2015 to April 2016, consecutive patients aged $\geq 18$ years, hospitalized with acute upper gastrointestinal bleeding, and endoscopically diagnosed with gastric and/or duodenal ulcers at Gia Dinh People's Hospital, Ho Chi Minh, Vietnam, were recruited prospectively. Patients with prior history of gastrectomy were excluded. The study protocol was approved by the Ethics Committee of Gia Dinh People's Hospital.

2.2. Data Collection. Patients admitted with the presentation of upper gastrointestinal bleeding (hematemesis, melena, or hematochezia) were resuscitated and prepared for upper gastrointestinal endoscopy. During endoscopy, the presence of blood in the upper gastrointestinal tract and the recent stigmata of bleeding ulcers were recorded. Endoscopic intervention was performed if high-risk lesions (i.e., spurting or oozing, visible vessels, or blood clot adhered to the ulcer base) were identified. When patients were stabilized after endoscopy, they were asked to fill out a questionnaire that included questions regarding demographic data, history of administration of nonsteroidal anti-inflammatory drugs (NSAIDs), proton pump inhibitors (PPIs), and antibiotics, peptic ulcer, and $H$. pylori eradication therapy. The hemodynamic instability at admission and preendoscopic PPI use were also recorded.

2.3. Helicobacter pylori Tests. H. pylori tests which were used in this study included rapid urease test (RUT), urea breath test (UBT), and serological and urinary $H$. pylori antibody tests. Patients without RUT or with negative RUT result then received noninvasive tests, which included UBT, and serological and urinary $H$. pylori antibody tests.
2.3.1. Rapid Urease Test. RUT (PyloriTek, Serim Research Corp., Elkhart, Ind., USA) was carried out randomly $(2: 1$ basis). Three biopsy specimens were taken and tested separately from each patient: the midantrum in the greater curvature (site 1), the lower corpus (site 2), and the midcorpus (site 3 ) in the greater curvature. The results were read after 1 hour as recommended by the manufacturer and considered to be positive when blue spots appeared over at least one specimen to the same color level as that of the positive control and negative when color changes were absent.

2.3.2. Urea Breath Test. UBT was performed once patients were allowed to drink and eat again. It was done using a commercially available diagnostic method (Helicobacter Test INFAI, INFAI GmbH, Cologne, Germany), with ${ }^{13} \mathrm{C}$ labelled urea to detect urease activity, indicating the presence of $H$. pylori $[13,14]$.

2.3.3. Serological Helicobacter pylori Antibody Test. Qualitative serological detection of specialized human immunoglobulin G (IgG) antibodies to H. pylori was done with InstantView ${ }^{\circledR}$ H. pylori Rapid Test (Alfa Scientific Designs Inc., Poway, CA, USA).

2.3.4. Urinary Helicobacter pylori Antibody Test. Urinary $H$. pylori antibody status was determined with a rapid urinary test (Rapirun ${ }^{\circledR}$ H. Pylori Antibody Stick, Otsuka Pharmaceutical Co., Ltd, Tokyo, Japan). The test measures human IgG antibody against $H$. pylori in urine using the principle of immunochromatography [10].

2.3.5. Definition of Helicobacter pylori-Positive. H. pylori infection was diagnosed when RUT was positive at any biopsy site or at least one other $H$. pylori test was positive.

2.4. Statistical Analysis. Baseline characteristics were presented as mean \pm standard deviation (SD) for continuous variables and as a frequency (percentage) for all variables. The recruited patients were divided into 2 groups according to the tests that used for $H$. pylori infection diagnosis: group A (RUT plus noninvasive $H$. pylori tests) and group B (only noninvasive $H$. pylori tests).

The chi-square and two-tailed Fisher's exact test were performed to evaluate whether the demographic, clinical characteristics, endoscopic findings, and H. pylori infection rate were different between groups $\mathrm{A}$ and $\mathrm{B}$. In addition, McNemar's test was used to assess the differences in $H$. pylori infection rates among different RUT biopsy sites.

\section{Results}

3.1. Patient Characteristics. During the study period, there were 177 consecutive patients with acute PUB admitted to Gia Dinh People's Hospital. Six patients with prior history of gastrectomy were excluded. The mean age of 171 patients recruited in the study was $55.4 \pm 17.3$. Forty-two $(24.6 \%)$ patients had prior history of gastroduodenal ulcers, including 
TABLE 1: Demographics and clinical characteristics $(n=171)$.

\begin{tabular}{lc}
\hline Demographics and clinical characteristics & $n(\%)$ \\
\hline Age (mean \pm SD) & $55.4 \pm 17.3$ \\
Male & $131(76.6)$ \\
Medical use before admission & \\
$\quad$ NSAIDs & $43(25.1)$ \\
$\quad$ PPIs $\leq 2$ weeks & $28(16.4)$ \\
$\quad$ Antibiotics $\leq 4$ weeks & $13(7.6)$ \\
Prior history of peptic ulcers & $42(24.6)$ \\
Prior history of H. pylori eradication & $12(7.0)$ \\
Presenting symptoms & \\
$\quad$ Hematemesis & $88(51.5)$ \\
$\quad$ Melena & $77(45)$ \\
$\quad$ Hematochezia & $6(3.5)$ \\
Preendoscopic PPIs use & $133(77.8)$ \\
$\quad$ High-dose, intravenous & $8(4.6)$ \\
Low-dose, intravenous & $3(1.8)$ \\
Oral & $27(15.8)$ \\
None & \\
Hemodynamic instability at admission (heart & $47(27.5)$ \\
rate $>100$ beats per minute and/or systemic & \\
blood pressure $<100$ mmHg) & \\
Transfusion requirement & \\
\hline
\end{tabular}

six with perforated peptic ulcer managed by simple suture operation. Twelve (7.0\%) patients had been diagnosed with $H$. pylori infection and received eradication therapy. Forty-three (25.1\%) patients used NSAIDs before admission. The number of patients using PPIs within 2 weeks and antibiotics within 4 weeks before admission was 28 (16.4\%) and 13 (7.6\%), respectively. Preendoscopy high-dose proton pump inhibitors were prescribed in $133(77.8 \%)$ patients. The detailed characteristics of patients in this study are presented in Table 1.

3.2. Endoscopic Setting and Findings. Upper gastrointestinal endoscopy was performed within 12 hours in 138 (80.7\%), within 12-24 hours in 16 (9.4\%), and within more than 24 hours after admission in 17 (9.9\%) patients. Blood was present in the gastrointestinal tract in $75(43.9 \%)$ patients. The proportions of gastric ulcer, duodenal ulcer, and gastroduodenal ulcer were $42.7 \%, 49.1 \%$, and $8.2 \%$, respectively. The highrisk stigmata of ulcer in this study included spurting/oozing $(11.7 \%)$, visible vessel $(14.6 \%)$, and adherent clot $(32.7 \%)$ (Table 2).

3.3. Helicobacter pylori Tests. RUT was performed in 111 patients (group A) and not performed in 60 patients (group B). No patients in group A required intervention for bleeding related to gastric mucosal biopsy for RUT. There were 8 patients in group A and 4 patients in group B who had prior history of $H$. pylori eradication therapy.

All patients with $H$. pylori infection in group A were diagnosed by RUT. Additional testing with serological and
TABLE 2: Endoscopic setting and findings $(n=171)$.

\begin{tabular}{lc}
\hline Endoscopic setting and findings & $n(\%)$ \\
\hline Timing & $138(80.7)$ \\
$<12 \mathrm{~h}$ & $16(9.4)$ \\
$12-24 \mathrm{~h}$ & $17(9.9)$ \\
$>24 \mathrm{~h}$ & $75(43.9)$ \\
Presence of blood in gastrointestinal tract & \\
Location of ulcer & $73(42.7)$ \\
Gastric & $84(49.1)$ \\
Duodenal & $14(8.2)$ \\
Gastric and duodenal & \\
Endoscopic stigmata & $1(0.6)$ \\
Spurting & $19(11.1)$ \\
Oozing & $25(14.6)$ \\
Visible vessel & $56(32.7)$ \\
Adherent clot & $17(9.9)$ \\
Red spot & $53(31.0)$ \\
Clean-based &
\end{tabular}

urinary tests and UBT identified no additional $H$. pylori infected patients (Figure 1). Patients without RUT or with negative RUT result then received noninvasive tests including serological and urinary tests and UBT. In group A, RUT was positive in $98.2 \%(109 / 111)$ patients including 6 of the 8 who had prior history of $H$. pylori eradication therapy. Two patients with negative RUT (both had no prior history of $H$. pylori eradication therapy) were tested with serological and urinary tests and UBT, which were all negative. In group B, 56 (93.3\%) patients without prior history of $H$. pylori eradication therapy were tested with serological and urinary tests and UBT, which showed 50 patients with $H$. pylori infection. Four (6.6\%) patients had prior history of $H$. pylori eradication therapy, and 2 of them were $H$. pylori-positive. $H$. pylori infection rate in group A was significantly higher than that in group B (98.2\% versus $86.7 \%, p=0.004)$ while the other characteristics between the 2 groups were not significantly different (Table 3).

The detection rates of $H$. pylori infection by RUT with each single biopsy taken from site $1(83.8 \%)$, site $2(90.1 \%)$, and site $3(85.6 \%)$ were not significantly different $(p=0.284)$ (Table 4 ). The detection rates by combined results from 2 biopsy sites: site 1 and site 2 (97.3\%), site 1 and site 3 (94.6\%), and site 2 and site 3 (95.5\%) were also not different from each other but significantly increased when compared with each single biopsy site (Figure 2). The detection rate combined result from 3 biopsy sites was the highest, 98.2\%.

\section{Discussion}

Accurately detecting $H$. pylori and subsequently eradicating the organism in infected patients with PUB are important to avoid recurrent bleeding. The recurrence rate has been reported in only $3 \%$ of the patients who received eradication 


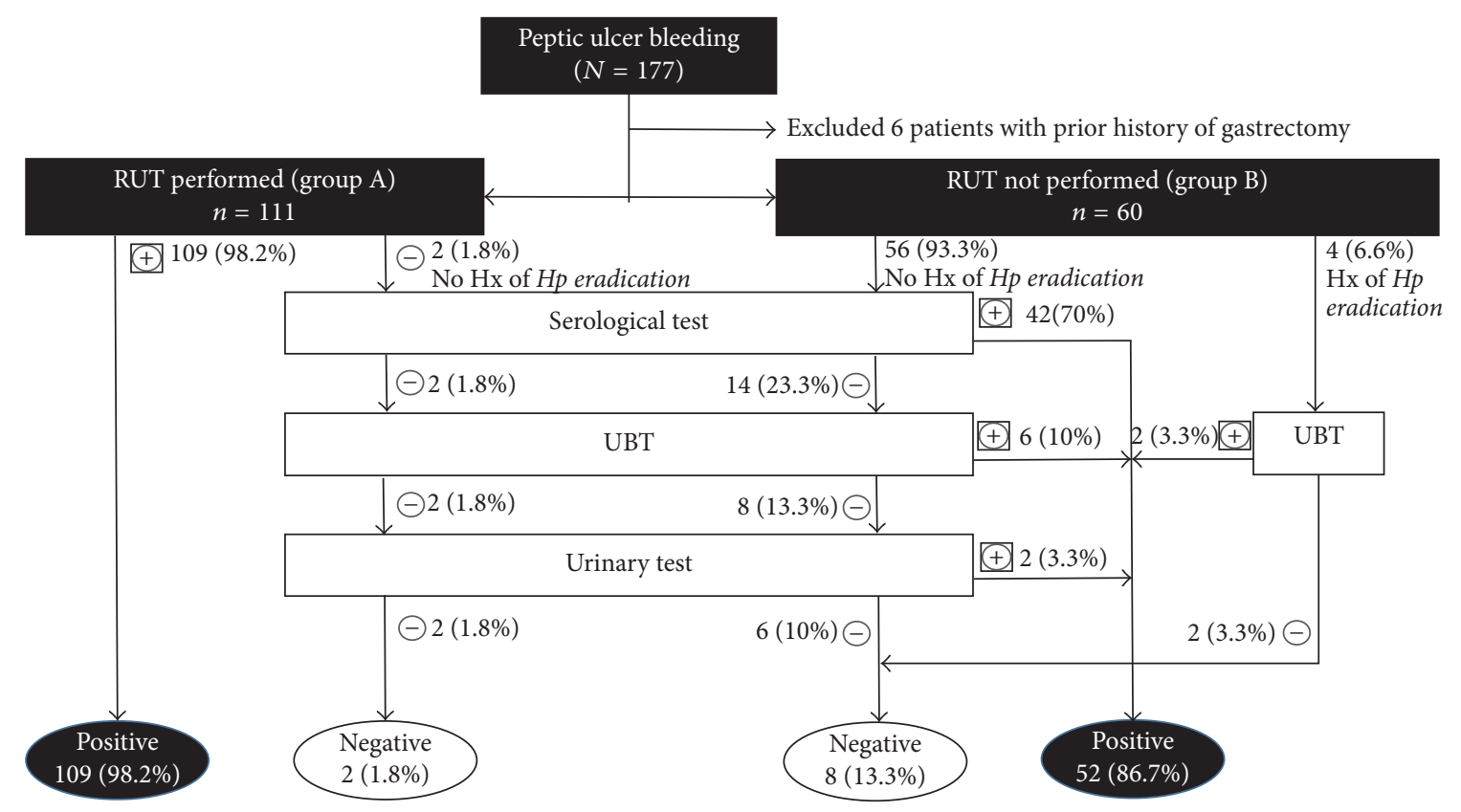

FIGURE 1: H. pylori testing in Vietnamese patients with acute peptic ulcer bleeding.

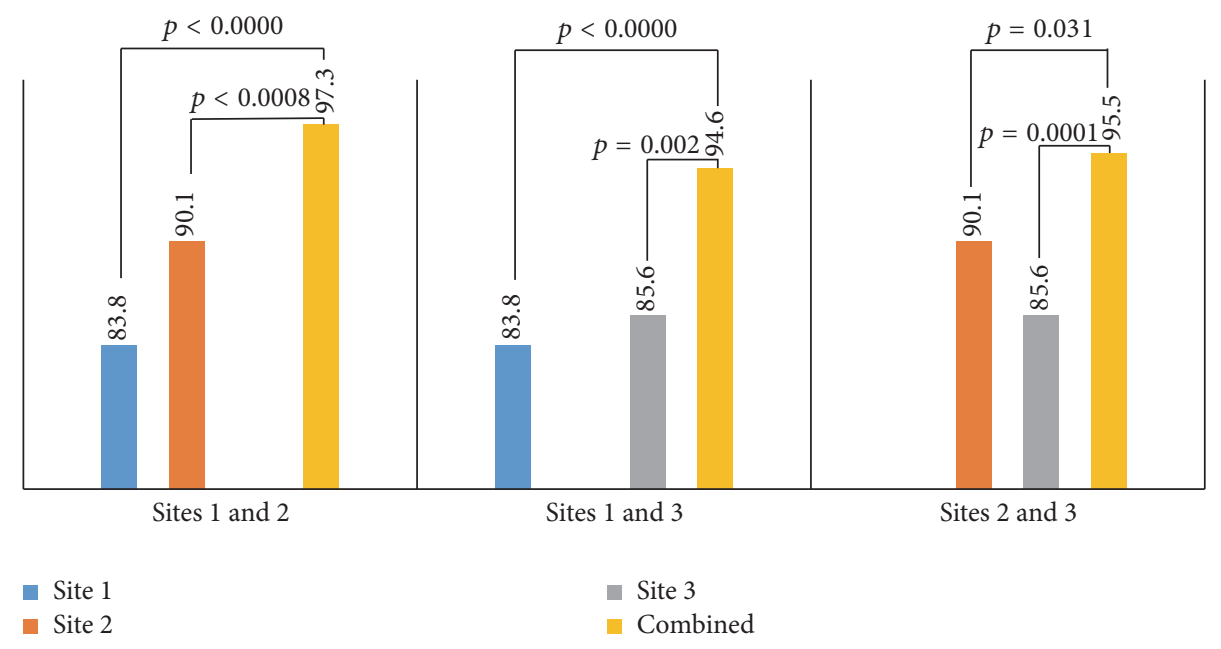

FIGURE 2: RUT results from single specimen versus combined specimens from two biopsy sites.

treatment but $20 \%$ in those treated with antisecretory noneradicating therapy (without subsequent long-term maintenance antisecretory therapy) $[15,16]$. The challenge is that many diagnostic tests including RUT, histology, and culture have been reported to have low sensitivity in patients with acute PUB [7]. In a metaregression analysis, the infection rate was significantly higher when diagnostic testing was delayed until at least 4 weeks following the bleeding event, suggesting that retesting $H$. pylori at a later time in PUB patients with initially negative test results was necessary [9]. However, late diagnosis of $H$. pylori infection in PUB patients leads to significant number of those with $H$. pylori infection who did not receive eradication therapy [4]. Therefore, increasing the sensitivity of tests for early diagnosis of $H$. pylori infection during admission period of PUB is crucial.
Regarding first-choice diagnostic tests for H. pylori, the American College of Gastroenterology recommended biopsy-based tests [17]. Among these, RUT is the most popular test in clinical practice but has high variable number of false-negative results according to a meta-analysis [7]. Sixteen studies included in this study showed a high degree of heterogeneity with sensitivities ranging between 0.41 and 0.94. When subanalysis of the biopsy sites for RUT was performed with only samples obtained from both the antrum and corpus which were considered, heterogeneity among sensitivities substantially decreased and pooled sensitivity increased. The results of our study help clarify this issue. The number and location of biopsy specimens are among key factors to increase RUT sensitivity. The detection rates of $H$. pylori infection by RUT with specimen taken from each 
TABLE 3: The characteristics of patients in groups $\mathrm{A}$ and $\mathrm{B}$.

\begin{tabular}{|c|c|c|c|}
\hline Characteristics & $\begin{array}{c}\text { Group A } \\
(n=111) \\
n(\%)\end{array}$ & $\begin{array}{c}\text { Group B } \\
(n=60) \\
n(\%)\end{array}$ & $p$ \\
\hline Age $($ mean \pm SD $)$ & $54.7 \pm 17.6$ & $56.1 \pm 16.8$ & 0.696 \\
\hline Male & $84(75.7)$ & $47(78.3)$ & 0.695 \\
\hline Prior history of gastroduodenal ulcers & $28(25.2)$ & $14(23.3)$ & 0.784 \\
\hline Prior history of $H$. pylori eradication & $8(7.2)$ & $4(6.7)$ & 1.000 \\
\hline \multicolumn{4}{|l|}{ Medical use before admission } \\
\hline NSAIDs & $28(25.2)$ & $15(25.0)$ & 0.974 \\
\hline PPIs within 2 weeks & $21(18.9)$ & $7(11.7)$ & 0.221 \\
\hline Antibiotics within 4 weeks & $10(9.0)$ & $3(5.0)$ & 0.547 \\
\hline $\begin{array}{l}\text { Hemodynamic instability at admission (heart rate }>100 \text { beats } \\
\text { per minute or systemic blood pressure }<100 \mathrm{mmHg} \text { ) }\end{array}$ & $29(26.1)$ & $18(30.0)$ & 0.219 \\
\hline \multicolumn{4}{|l|}{ Preendoscopic PPIs use } \\
\hline High-dose, intravenous & $89(80.2)$ & $44(73.3)$ & \multirow{4}{*}{0.136} \\
\hline Low-dose, intravenous & $6(5.4)$ & $2(3.3)$ & \\
\hline Oral & $3(2.7)$ & $0(0)$ & \\
\hline None & $13(11.7)$ & $14(23.3)$ & \\
\hline \multicolumn{4}{|l|}{ Timing of endoscopy } \\
\hline$<12 \mathrm{~h}$ & $84(75.7)$ & $54(90.0)$ & \multirow{3}{*}{0.077} \\
\hline $12-24 \mathrm{~h}$ & $13(11.7)$ & $3(5.0)$ & \\
\hline$>24 \mathrm{~h}$ & $14(12.6)$ & $3(5.0)$ & \\
\hline Presence of blood in endoscopy & $52(46.8)$ & $23(38.3)$ & 0.284 \\
\hline \multicolumn{4}{|l|}{ Location of ulcer } \\
\hline Gastric & $49(44.1)$ & $24(40.0)$ & \multirow{3}{*}{0.763} \\
\hline Duodenal & $54(48.6)$ & $30(50.0)$ & \\
\hline Gastric and duodenal & $8(7.2)$ & $6(10.0)$ & \\
\hline \multicolumn{4}{|l|}{ Endoscopic stigmata } \\
\hline Spurting & $1(0.9)$ & $0(0)$ & \multirow{6}{*}{0.448} \\
\hline Oozing & $13(11.7)$ & $6(10.0)$ & \\
\hline Visible vessel & $20(18.0)$ & $5(8.3)$ & \\
\hline Adherent clot & $33(29.7)$ & $23(38.3)$ & \\
\hline Red spot & $12(10.8)$ & $5(8.3)$ & \\
\hline Clean-based & $32(28.8)$ & $21(35.0)$ & \\
\hline H. pylori-positive rates & $109(98.2)$ & $52(86.7)$ & 0.004 \\
\hline
\end{tabular}

biopsy site in our study were not different, but significantly increased when specimens from 2 biopsy sites were combined (Table 4 and Figure 2). The combined result from 3 biopsy sites helps to detect even more patients with $H$. pylori infection, showing that negative RUT result from 2 biopsy sites is still not enough to exclude $H$. pylori infection and additional diagnostic tests or delaying diagnostic tests should be done.

Because some studies have found that all endoscopicbased diagnostic tests have a lower sensitivity in patients with acute PUB [7], it has been recommended that noninvasive methods should be used in patients who have negative result of endoscopic-based diagnostic tests [17]. Two patients in group A who had negative RUT result were performed serological test, UBT, and urinary test and had negative results. Therefore, all patients with $H$. pylori infection in group A were diagnosed by RUT and the total infection rate in this group was $98.2 \%$. As the specificity of RUT during PUB is very high $[6,7]$, this figure likely represents for the true prevalence of $H$. pylori in Vietnamese patients with PUB. This is truly first data on Vietnamese population so far. Previous studies in other populations showed a significant lower prevalence of $H$. pylori in PUB patients. In European studies, the prevalence of $H$. pylori in PUB patients was lower than that in patients with uncomplicated peptic ulcer disease, varying from $43 \%$ to $56 \%$, and was explained by NSAIDs use [18]. In our study, the rate of NSAIDs use was $25.1 \%$. In addition to this, a significant number of patients had prior history of NSAIDs use and were also infected with $H$. pylori. These 2 factors have been confirmed as independent risk 
TABLE 4: RUT results with specimens taken from different biopsy sites.

\begin{tabular}{lc}
\hline Biopsy sites & $\begin{array}{c}\text { H. pylori-positive cases } \\
n(\%)\end{array}$ \\
\hline Single biopsy site & \\
1 (midantrum, greater curvature) & $93(83.8)$ \\
2 (low-corpus, greater curvature) & $100(90.1)$ \\
3 (midcorpus, greater curvature) & $95(85.6)$ \\
Two biopsy sites (combined results) & \\
1 \& 2 & $108(97.3)$ \\
$1 \& 3$ & $105(94.6)$ \\
2 \& 3 & $106(95.5)$ \\
Three biopsy sites (combined result) & \\
1,2 , and 3 & $109(98.2)$ \\
\hline
\end{tabular}

factors for the development of peptic ulcer disease, associated bleeding, and the risk was significantly augmented when both factors presented [19]. Therefore, patients with PUB who have a single negative $H$. pylori test and a prior history of NSAIDs use should not be simply referred to as NSAIDs-induced PUB. Further diagnostic tests should be done to detect $H$. pylori infection.

The difference in infection rate of $H$. pylori between our study and other studies may be explained by many reasons, such as the different infection rate of $H$. pylori in each population, the differences in biopsy protocol for RUT, the endoscopy timing, and the types of RUT kit among studies. Many other studies used CLOtest ${ }^{\circledR}$ while we used PyloriTek ${ }^{\circledR}$ $[6,7]$. Previous studies showed that results of the PyloriTek at 1 hour and CLOtest at 24 hours are comparable [20, 21], but there have been no direct comparison of the 2 kits in the setting of PUB patients, which is a research question of our future study.

Although biopsy-based tests for H. pylori are recommended as preferred diagnostic tests in PUB patients, they could not be performed in some situations because endoscopic treatment may have already made the procedure too long or patients were not hemodynamically stable during the procedure. In some developing countries, upper gastrointestinal endoscopy is performed under local anesthesia and patients' cooperation with prolonged procedure may be difficult. Therefore, noninvasive tests for $H$. pylori diagnosis are still required. According to a meta-analysis, $H$. pylori infection was accurately diagnosed by serological test in patients with PUB [7]. In our study, the test was the first choice among noninvasive diagnostic tests as it is widely available. In addition, UBT was used if serological test was negative. And urinary test was also used as it was locally validated in Vietnam with acceptable accuracy [10]. Although the specificity of this noninvasive approach is not as good as that of RUT in the setting of recent gastrointestinal bleeding [7], we try to combine this set of noninvasive tests in order to early detect all possibly $H$. pylori infection during admission time. But in spite of combining these three noninvasive tests, the total positive rate of $H$. pylori infection in group
B was still significantly lower than that in group A. As other characteristics between the 2 groups were not different (Table 3), this result clearly shows that RUT with at least 2 biopsy sites is an important test for $H$. pylori diagnosis in patients with PUD.

Our study has several weak points. First, number of patients included in this study was relatively limited. Studies with much more patients are needed to verify our results. Second, the definition of $H$. pylori-positive was based on only one diagnostic test. One false-positive finding may affect the diagnosis. And third, diagnostic tests for $H$. pylori infection such as histological, cultural, and fecal antigen tests were not included in the design of this study. If these tests were included in this study, the results might differ.

In conclusion, our study shows that the H.pylori infection rate in Vietnamese patients with acute PUB is high. RUT is an excellent test for detecting $H$. pylori infection in this setting if at least 2 specimens from different biopsy sites are taken. In case that RUT is not performed, late H. pylori retesting may be required even when a combined set of noninvasive tests have shown negative results.

\section{Competing Interests}

The authors declare that they have no competing interests.

\section{Authors' Contributions}

Dr. Naomi Uemura took responsibility for the integrity of the work as a whole, from inception to published article. Duc Trong Quach, Toru Hiyama, and Shinji Tanaka designed the research study. Duc Trong Quach, Mai Ngoc Luu, ThuyHuongThi To, Quy Nhuan Bui, Binh Duy Tran, and MinhCong Hong Vo performed the research and collected data. Mai Ngoc Luu and Duc Trong Quach analysed the data. Duc Trong Quach, Toru Hiyama, and Naomi Uemura wrote the paper. All authors approved the final version of the manuscript.

\section{Acknowledgments}

The authors thank Drs. Phong Ha and Hung Le for their kindly advice to prepare the manuscript.

\section{References}

[1] J. P. Gisbert, X. Calvet, F. Feu et al., "Eradication of Helicobacter pylori for the prevention of peptic ulcer rebleeding," Helicobacter, vol. 12, no. 4, pp. 279-286, 2007.

[2] J. P. Gisbert, X. Calvet, A. Cosme et al., "Long-term follow-up of 1,000 patients cured of Helicobacter pylori infection following an episode of peptic ulcer bleeding," The American Journal of Gastroenterology, vol. 107, no. 8, pp. 1197-1204, 2012.

[3] S. S. Chang and H.-Y. Hu, "Helicobacter pylori eradication within 120 days is associated with decreased complicated recurrent peptic ulcers in peptic ulcer bleeding patients," Gut and Liver, vol. 9, no. 3, pp. 346-352, 2015.

[4] H. Yoon, D. H. Lee, E. S. Jang et al., "Optimal initiation of Helicobacter pylori eradication in patients with peptic ulcer 
bleeding," World Journal of Gastroenterology, vol. 21, no. 8, pp. 2497-2503, 2015.

[5] J. J. Kim, J. S. Lee, S. Olafsson, and L. Laine, "Low adherence to helicobacter pylori testing in hospitalized patients with bleeding peptic ulcer disease," Helicobacter, vol. 19, no. 2, pp. 98-104, 2014.

[6] Y. J. Choi, N. Kim, J. Lim et al., "Accuracy of diagnostic tests for Helicobacter pylori in patients with peptic ulcer bleeding," Helicobacter, vol. 17, no. 2, pp. 77-85, 2012.

[7] J. P. Gisbert and V. Abraira, "Accuracy of Helicobacter pylori diagnostic tests in patients with bleeding peptic ulcer: a systematic review and meta-analysis," American Journal of Gastroenterology, vol. 101, no. 4, pp. 848-863, 2006.

[8] J.-H. Tang, N.-J. Liu, H.-T. Cheng et al., "Endoscopic diagnosis of Helicobacter pylori infection by rapid urease test in bleeding peptic ulcers: a prospective case-control study," Journal of Clinical Gastroenterology, vol. 43, no. 2, pp. 133-139, 2009.

[9] J. Sánchez-Delgado, E. Gené, D. Suárez et al., "Has H. pylori prevalence in bleeding peptic ulcer been underestimated? a meta-regression," American Journal of Gastroenterology, vol. 106, no. 3, pp. 398-405, 2011.

[10] D. T. Quach, T. Hiyama, F. Shimamoto et al., "Value of a new stick-type rapid urine test for the diagnosis of Helicobacter pylori infection in the Vietnamese population," World Journal of Gastroenterology, vol. 20, no. 17, pp. 5087-5091, 2014.

[11] T. L. Nguyen, T. Uchida, Y. Tsukamoto et al., "Helicobacter pylori infection and gastroduodenal diseases in Vietnam: a cross-sectional, hospital-based study," BMC Gastroenterology, vol. 10, article no. 114, 2010.

[12] P. Malfertheiner, F. Megraud, C. A. O’Morain et al., "Management of Helicobacter pylori infection-the Maastricht IV/Florence Consensus Report," Gut, vol. 61, no. 5, pp. 646-664, 2012.

[13] K. E. L. McColl, L. S. Murray, D. Gillen et al., "Randomised trial of endoscopy with testing for Helicobacter pylori compared with non-invasive $\mathrm{H}$ pylori testing alone in the management of dyspepsia," British Medical Journal, vol. 324, no. 7344, pp. 999$1002,2002$.

[14] H. Alberti, "Gastro-oesophageal reflux disease in general practice. Utility and acceptability of Infai C13-urea breath test has been shown," British Medical Journal, vol. 324, no. 7335, pp. 485486, 2002.

[15] J. P. Gisbert, S. Khorrami, F. Carballo, X. Calvet, E. Gené, and J. E. Dominguez-Muñoz, "H. pylori eradication therapy vs. antisecretory non-eradication therapy (with or without longterm maintenance antisecretory therapy) for the prevention of recurrent bleeding from peptic ulcer," The Cochrane Database of Systematic Reviews, no. 2, Article ID CD004062, 2004.

[16] J. P. Gisbert, S. Khorrami, F. Carballo, X. Calvet, E. Gene, and E. Dominguez-Muñoz, "Meta-analysis: Helicobacter pylori eradication therapy vs. antisecretory non-eradication therapy for the prevention of recurrent bleeding from peptic ulcer," Alimentary Pharmacology and Therapeutics, vol. 19, no. 6, pp. 617-629, 2004.

[17] L. Laine and D. M. Jensen, "Management of patients with ulcer bleeding," American Journal of Gastroenterology, vol. 107, no. 3, pp. 345-360, 2012.

[18] M. E. van Leerdam, "Epidemiology of acute upper gastrointestinal bleeding," Best Practice and Research: Clinical Gastroenterology, vol. 22, no. 2, pp. 209-224, 2008.

[19] J.-Q. Huang, S. Sridhar, and R. H. Hunt, "Role of Helicobacter pylori infection and non-steroidal anti-inflammatory drugs in peptic-ulcer disease: a meta-analysis," The Lancet, vol. 359, no. 9300, pp. 14-22, 2002.

[20] T. Puetz, N. Vakil, S. Phadnis, B. Dunn, and J. Robinson, “The Pyloritek test and the CLO test: accuracy and incremental cost analysis," American Journal of Gastroenterology, vol. 92, no. 2, pp. 254-257, 1997.

[21] Y. Elitsur, I. Hill, S. N. Lichtman, and A. J. Rosenberg, "Prospective comparison of rapid urease tests (PyloriTek, CLO test) for the diagnosis of Helicobacter pylori infection in symptomatic children: A Pediatric Multicenter Study," American Journal of Gastroenterology, vol. 93, no. 2, pp. 217-219, 1998. 


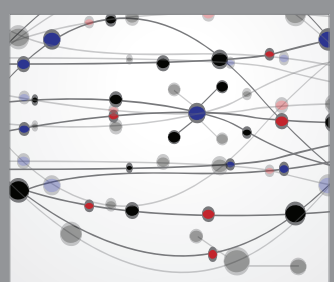

The Scientific World Journal
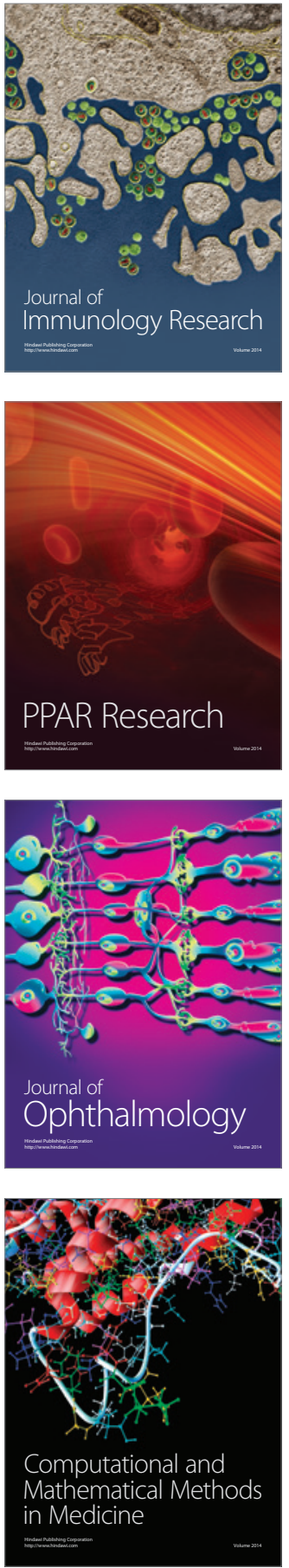

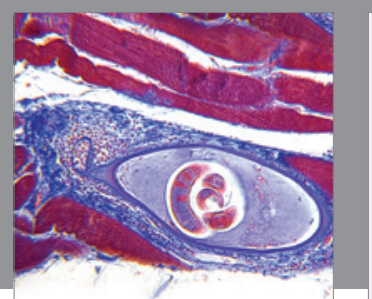

Gastroenterology Research and Practice
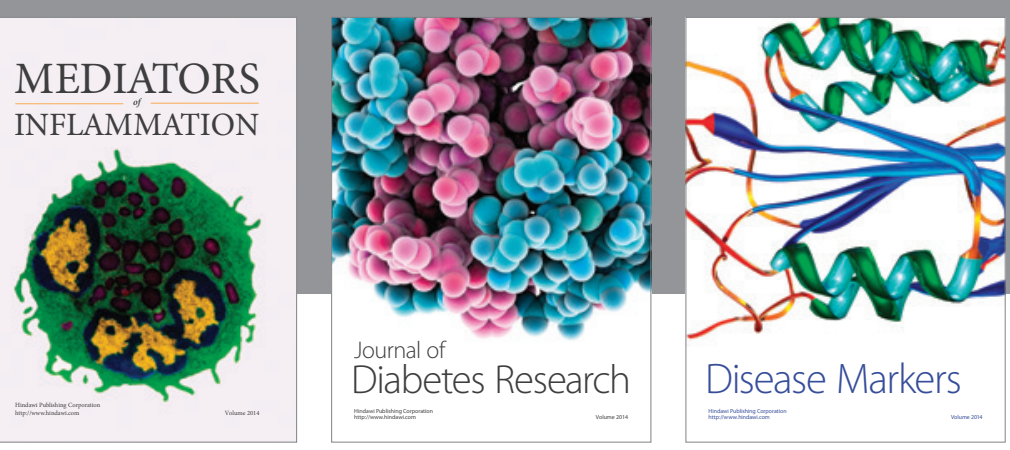

Disease Markers

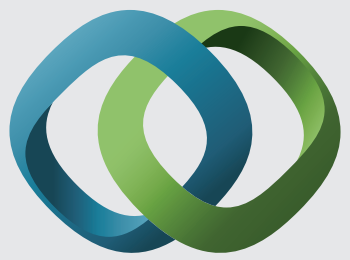

\section{Hindawi}

Submit your manuscripts at

https://www.hindawi.com
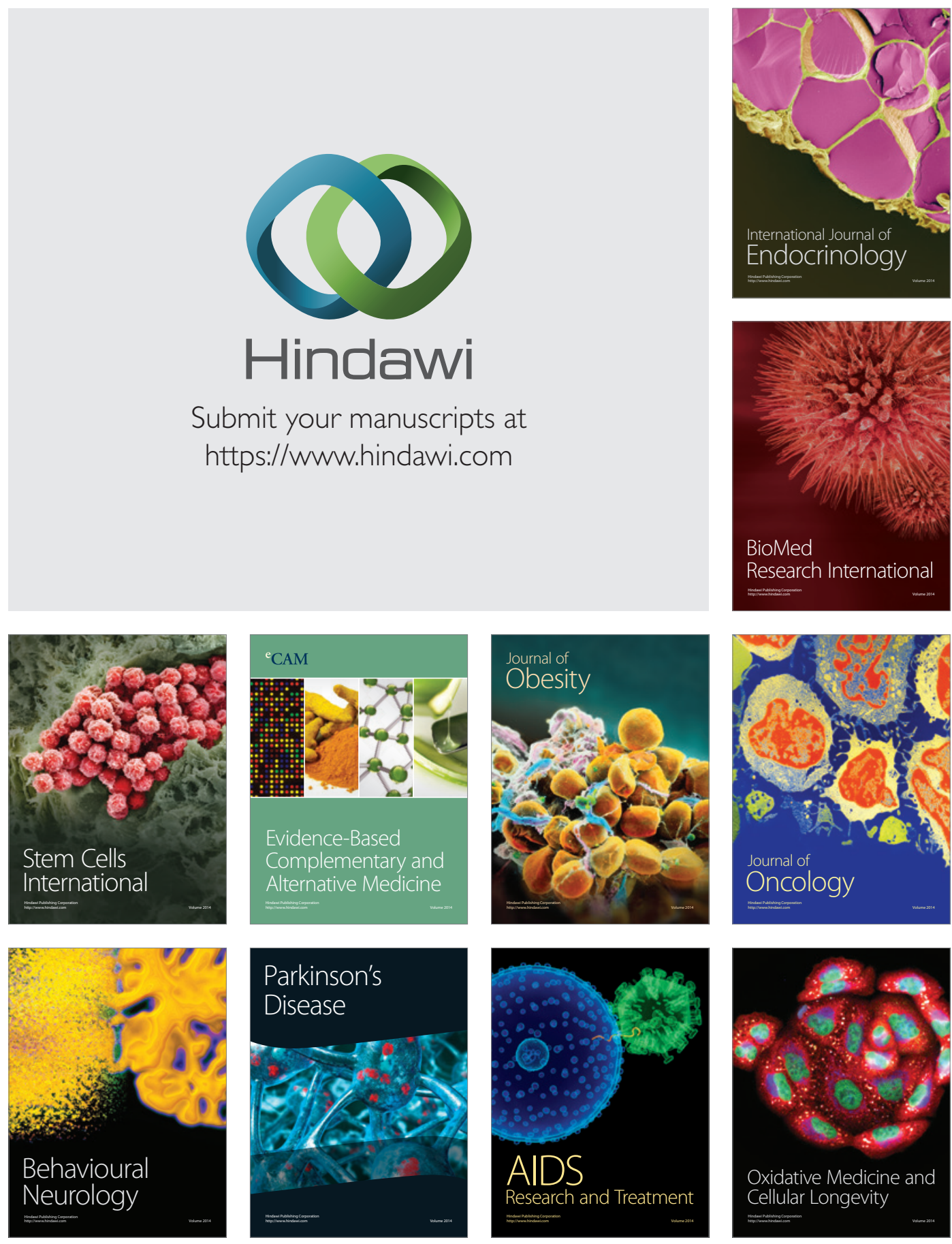